\title{
STRUCTURE OF THE TURBULENCE IN PULSATING PIPE FLOWS
}

\author{
TOKURO MIZUSHINA, TOSHIRO MARUYAMA* \\ AND HIDEO HIRASAWA ${ }^{1}$ \\ Department of Chemical Engineering, Kyoto University, Kyoto, 606
}

\begin{abstract}
An experimental investigation was performed to study the dynamic process of bursting in pulsating turbulent flow. Particular emphasis is placed on the details of the process of turbulence generation and its propagation in the radial direction.

The results show that the resonance in pulsating flow affects only the generation of turbulence, and that another important factor which characterizes the dynamic behaviour of turbulence is the coherency of propagation of generated turbulence: changing its frequency, the generated turbulence propagates to the centre-line of the tube with a unique propagation time which scales on the wall parameters. The propagation time in which the turbulence propagates from the position of origin to the centre-line agrees well with the mean burst period.

This fact suggests that the entire cycle of the bursting phenomenon is characterized by the propagation of generated turbulence in the radial direction and that the bulk parameter dependence of burst period is attributable to the pipe radius.
\end{abstract}

\section{Introduction}

In steady turbulent flow, the importance of the wall region in transport phenomena and the generation and maintenance of turbulence is well known.

Recently, visual studies have shed new light on the study of the turbulence structure of the wall region. Applying a combined dye-injection and hydrogenbubble technique to an artifically-tripped turbulent boundary layer, Kline et al. ${ }^{2)}$ observed that the wall area $\left(0<y^{+}<100\right)$ showed a distinct pattern characterized by a deterministic sequence of events occurring randomly in space and time. They described the sequence as being composed of three stages: (i) the appearance of a relatively low-speed region of fluid near the wall; (ii) the 'lift-up' of this 'low-speed streak' from the wall followed by some form of 'oscillatory growth' and (iii) ultimately, the 'breakup' of any sign of coherency in the visual representations of this structure. This entire three-stage process was called 'bursting'. From estimates made from the hydrogenbubble data, they observed that practically all the turbulence production occurred during bursting. In addition, they strongly suggested that the cycle was intermittent but had a definite preferred range of period of occurrence and a well-defined mean period, i. e. mean burst period.

Corino and Brodkey ${ }^{1}$, using a dark-field illumination technique in successive strips of the flow, observed

Received October 28, 1974.

Presented in part at the 8 th Autumn Meeting of The Soc.

Chem. Engrs., Japan, Oct. 9, 1974.

1 Shin-etsu Chemical Co., Ltd. a deterministic sequence of events which are in essential agreement with those reported by Kline, et al. They suggested that an interaction between accelerated and retarded flow was fundamental to the lift-up process.

Rao et al. ${ }^{8)}$ measured the burst period over a wide Reynolds number range using a hot-wire anemometry technique in a turbulent boundary layer. Their measurements indicated that the mean burst period scaled with the variables of the outer flow instead of the inner variables. Moreover, according to Kline et $a l .^{2}$, the time period of the observed bursting process near the wall is only a part of the whole burst period. Therefore, a sequence of events subsequent to the breakup is imagined to occur in the core region.

More recently, Nychas et al. ${ }^{5)}$ and Offen and Kline ${ }^{6)}$, using the same visual techniques as those used by Corino and Brodkey and by Kline et al., respectively, observed the outer region of turbulent boundary layer and suggested that the transverse vortex was a part of the bursting process. However, it seems very difficult to make clear the entire cycle of events of the bursting phenomenon in steady turbulent flow, because the generation of turbulence near the wall occurs randomly in space and time.

On the other hand, as was clarified in a previous paper ${ }^{4}$, the bursting phenomenon in pulsating flow shows the same periodicity as the pulsation period, i. e. resonance occurs, if the pulsation period is within a preferred range of burst period observed in steady flow. It is expected that detailed study of spatial and temporal relation between events in resonant pulsating flow will show the entire cycle of events which characterizes the periodicity of bursting. Thus study 
of resonant pulsating flow is worth while.

First, at constant Reynolds number $\left(R e=10^{4}\right)$ some characteristics of resonance were examined in the whole range of burst period. Next, turbulence generation and its propagation in the radial direction were made clear by using a phase-averaged correlation coefficient. In particular, detailed studies were made of the propagation process and its time period. Finally, a relation between burst period and propagation time was studied.

\section{Experiments}

The experiments were conducted in a fully developed pipe flow with a periodical pulsation of flow rate produced by means of two bellows pumps. The instantaneous velocity was measured by using the electrochemical method.

A complete description of the experimental apparatus has been given in previous papers ${ }^{3,4)}$. These include the fluid, the test section, the mass transfer probe and the electronic equipment. In the present work, the same apparatus was used for the bulk of the measurements. For short pulsation period, however, measurements were made in a tube of $5.16 \mathrm{~cm}$ inside diameter, at a position 78 diameters downstream from the inlet.

The analog-to-digital conversion and data reduction were performed with a hybrid computer using several assembly language programmes. To avoid too long recording time and difficulty of adjusting an external clock to the pulsation period, the electrical signals of velocity and time pulse were directly connected to the analog input. The time pulse was generated by a photo-transistor at 32 phase angles per cycle according to the flow pulsation.

\section{Results and Discussion}

\section{1 Region of burst period in steady flow}

To confirm a flow being fully developed in the tube of $5.16 \mathrm{~cm}$ inside diameter, turbulence characteristics in steady flow were measured first. Figs. 1 and 2 show the turbulence intensity profile and the mean burst period $\overline{T_{B}}$, respectively. The mean burst period was determined from the delay time required to obtain the second mild maximum in the curve of the autocorrelation coefficient ${ }^{4)}$. They are in sufficiently good agreement with the measurements in the tube of $2 \mathrm{~cm}$ inside diameter and those of other investigators ${ }^{7}$.

The minimum burst period $T_{B_{m i n}}$ is also shown in Fig. 2, which was obtained by the same method as that used in the previous paper ${ }^{4}$. As was pointed out in that paper, the value of $T_{B_{m i n}}$ is nearly symmetric to the maximum burst period $T_{c}$ with respect to $\overline{T_{B}}$ on a $\log$ scale.

The following sections will discuss pulsating flow

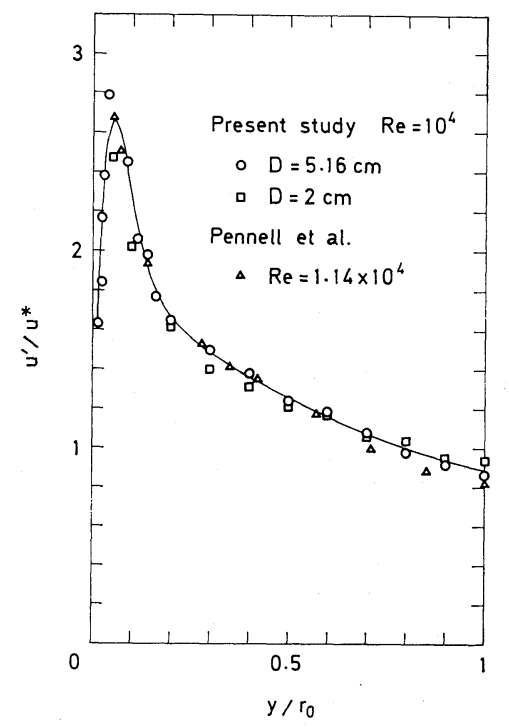

Fig. 1 Turbulence intensity profile in steady flow

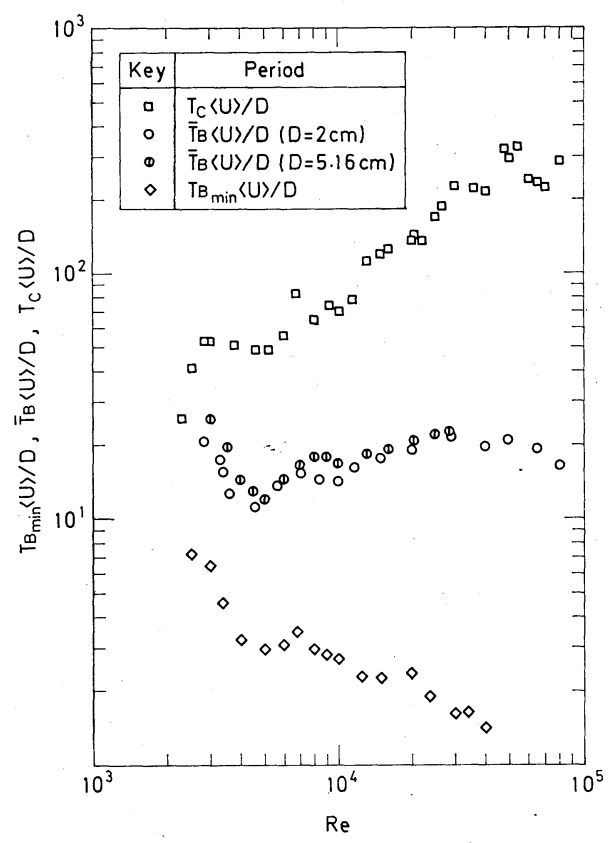

Fig. $2 T_{c}, \overline{T_{B}}$ and $T_{B \min }$ normalized with bulk parameters as functions of Reynolds number

of pulsation period $T$ within the preferred range of burst period, i. e. $T_{B_{m i n}}<T<T_{c}$.

\section{2 Resonant pulsating flow}

At various periods of pulsation ranging from $T_{B_{m i n}}$ to $\overline{T_{B}}$ the velocity and turbulence intensity profiles were measured in the circular tube of $5.16 \mathrm{~cm}$ inside diameter. The phase average was made at 32 phase angles within one cycle by using the instantaneous values of 100 pulsation cycles:

for velocity

$$
U(t)=\frac{1}{N} \sum_{n=1}^{N} U_{i}(t+n T)
$$

and for turbulence intensity 


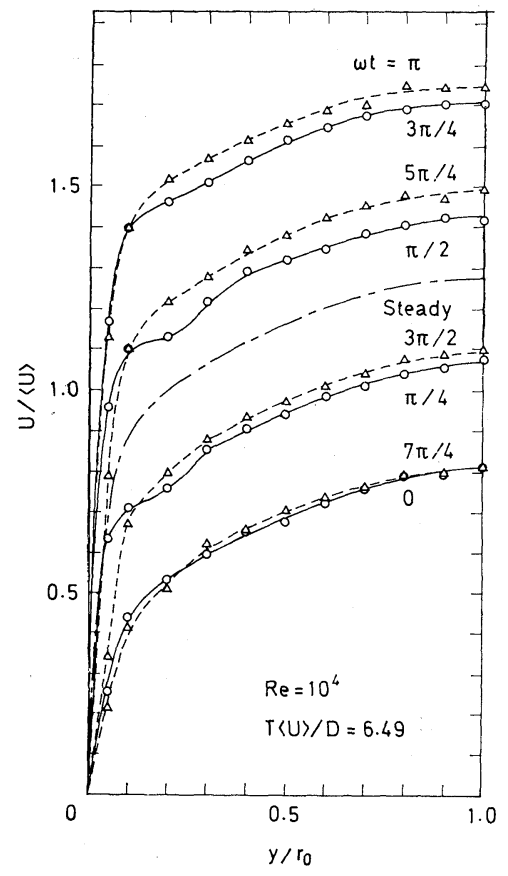

Fig. 3 Velocity profiles in pulsating flow
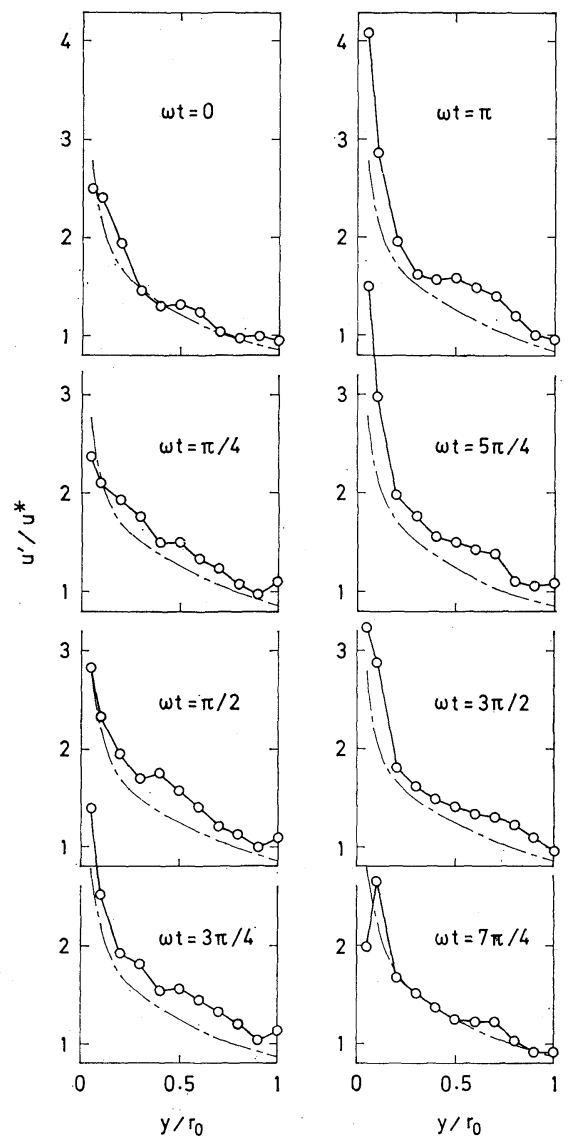

$\operatorname{Re}=10^{4}$

$\mathrm{T}\langle U\rangle / \mathrm{D}=6.49$ -.- Steady

Fig. 4 Turbulence intensity profiles in pulsating flow

$$
u^{\prime}(t)=\sqrt{\frac{1}{N} \sum_{n=1}^{N}\left\{U_{i}(t+n T)-U(t)\right\}^{2}}
$$

where $N=100$.

From the measurements, it was clarified that the

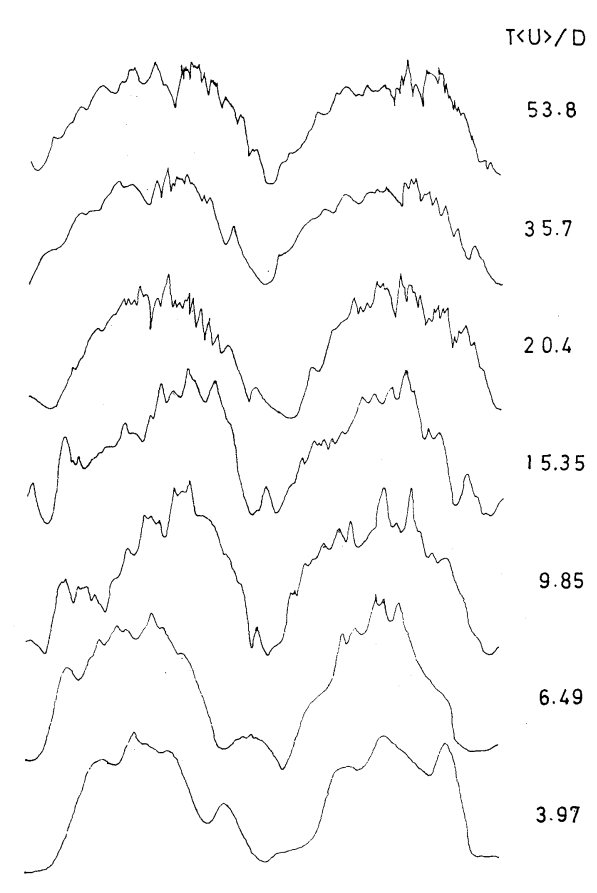

$R e=10^{4} \quad y / r_{0}=0.05$

Fig. 5 Variations of velocity $\left(\boldsymbol{T}_{B_{\min }}<\boldsymbol{T}<\boldsymbol{T}_{\boldsymbol{c}}\right)$

resonance between pulsation and turbulence generation occurred in the whole region of $T_{B_{m i n}}<T<T_{c}$. In addition, it was found that the characteristics of resonance which were pointed out in the previous paper $^{4}$ became more obscure with decreasing pulsation period. These details will next be described.

Fig. 3 shows a typical set of velocity profiles. The parameter varied in this diagram is the phase angle $\omega t$ (started from the time when the flow rate had the minimum value). The velocity profiles in pulsating flow are similar to those of steady flow with the exception that the profiles near the wall are somewhat contorted in the acceleration period.

Fig. 4 shows normalized profiles of intensity of turbulence. The friction velocity $u^{*}$ was determined in the steady-flow condition. Near the wall they vary around the profile of steady flow, and rise considerably at about $\omega t=\pi$. This violent change of intensity of turbulence is apparently caused by the resonance. In the core region of the tube, however, these profiles are different from Profiles II mentioned in the previous paper $^{4)}$ and similar to those for steady flow.

The area in which the similarity persists extends near to the wall continuously as pulsation period decreases from $\overline{T_{B}}$ to $T_{B_{\text {min }} \text {. }}$ Fig. 5 illustrates several examples of velocity signal at $y / r_{0}=0.05$. The periodicity of turbulent fluctuation appears distinctly at long pulsation period $(T\langle U\rangle \mid D \geqslant 20.4)$ but it becomes obscure with decreasing pulsation period.

The above-mentioned facts strongly suggest that the pulsation affects only the generation of turbulence, 


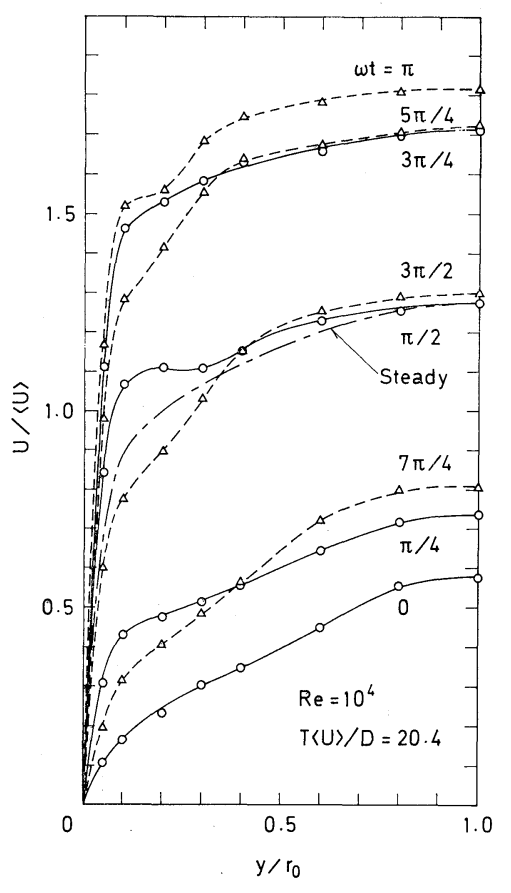

Fig. 6 Velocity profiles in pulsating flow

and that another factor rules the bursting process subsequent to the generation of turbulence. Detailed explanation in the following section uses a correlation function measured in the region of $\overline{T_{B}}<T<T_{c}$, where the periodical change of turbulence is detectable in the core region of the tube.

\section{3 Phase-averaged correlation coefficient}

The correlation function for $u, R_{u u}\left(t, t^{\prime}\right)$ is defined by the equation

$$
R_{u u}\left(t, t^{\prime}\right)=\frac{\overline{u(t) u\left(t+t^{\prime}\right)}}{u^{\prime}(t) u^{\prime}\left(t+t^{\prime}\right)}
$$

where $t^{\prime}$ is delay time and $u^{\prime}(t)$ is not equal to $u^{\prime}\left(t+t^{\prime}\right)$ except when $t^{\prime}$ is equal to $T$, because the turbulence intensity varies with respect to phase. An overline in Eq. (3) means the phase average, and so Eq. (3) is rewritten as

$$
R_{u u}\left(t, t^{\prime}\right)=\frac{\sum_{n=1}^{N} u(t+n T) u\left(t+n T+t^{\prime}\right)}{\sqrt{\sum_{n=1}^{N}\{u(t+n T)\}^{2} \sum_{n=1}^{N}\left\{u\left(t+n T+t^{\prime}\right)\right\}^{2}}}
$$

where $N=100$ and $-T / 2 \leqslant t^{\prime} \leqslant T / 2$.

Measurements were made at $T=0.764 \mathrm{sec}$ by using the tube of $2 \mathrm{~cm}$ inside diameter. Under this condition, both profiles of velocity and turbulence intensity shown in Figs. 6 and $\mathbf{7}$ are similar to Profiles $\mathrm{II}^{4)}$.

Fig. 8 shows the correlation curves at $y / r_{0}=0.05$ or $y^{+}=15.7$, where the generation of turbulent energy near the wall is supposed to be detected. As can be seen, there occurs a periodical change of time scale: a large scale of turbulence decreases during the
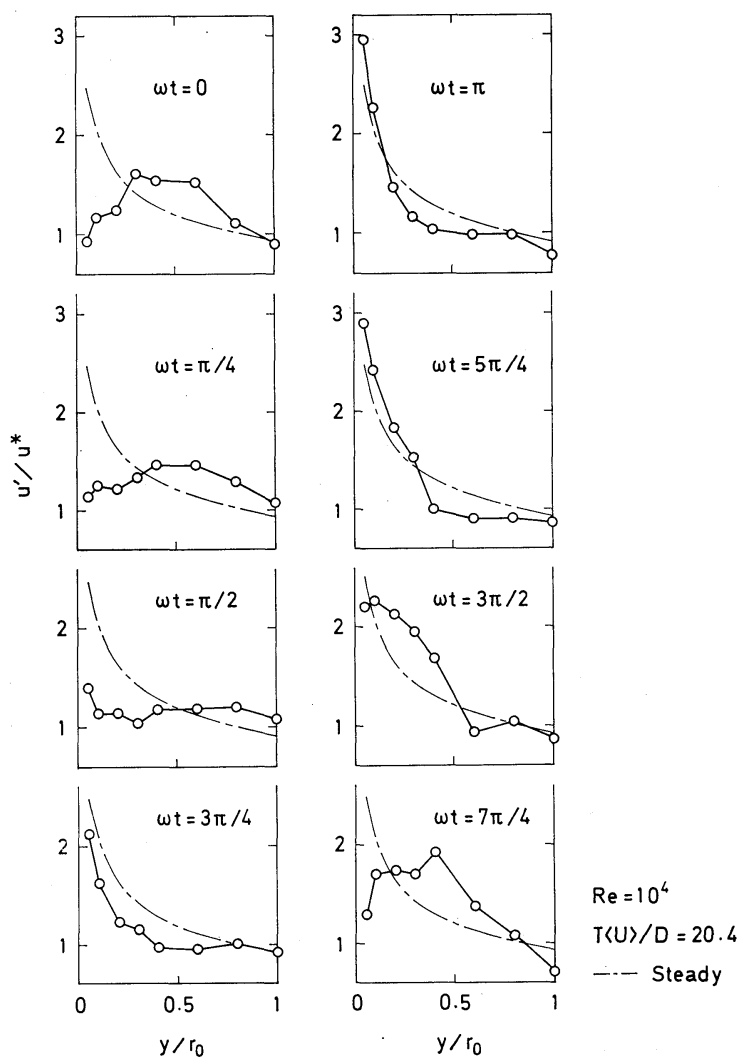

Fig. 7 Turbulence intensity profiles in pulsating flow
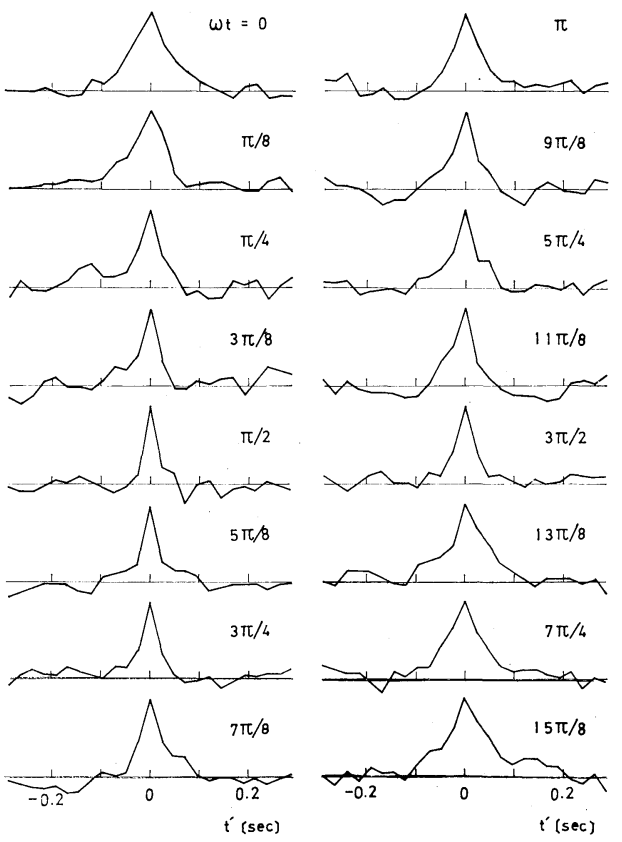

$\operatorname{Re}=10^{4} \quad T\langle U\rangle / D=20.4$

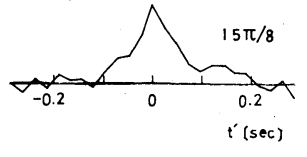

8 Autocorrelation coefficients in pulsating flow

phase $0<\omega t<\pi / 2$ and increases slowly during $\pi / 2<\omega t<2 \pi$. Note that they are not always symmetric with respect to the axis of $t^{\prime}=0$, although an usual correlation curve in steady flow is assumed to be always symmetric, i. e. an even function of delay time. In particular, a distinct asymmetry at $\omega t=\pi / 4$ indi- 


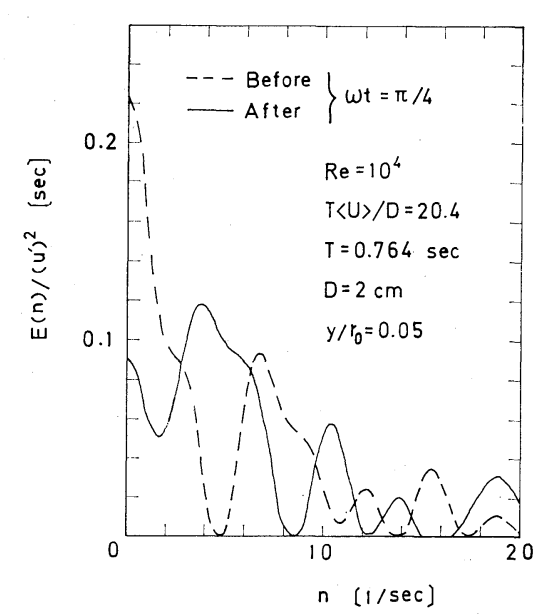

Fig. 9 One-dimensional energy spectra just before and after phase angle $\pi / 4$

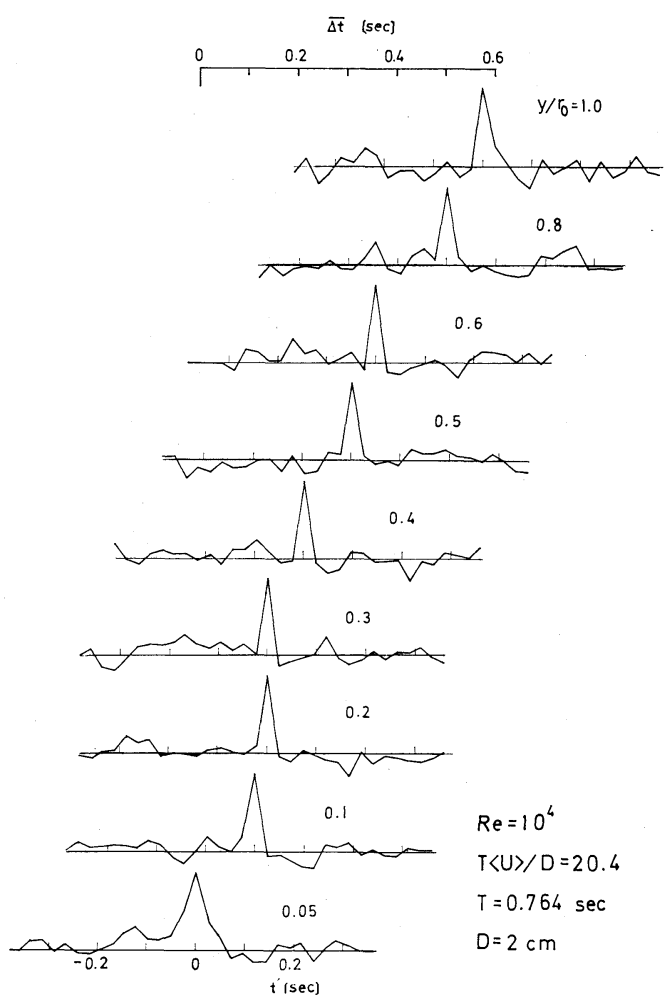

Fig. 10 Radial distribution of asymmetrical autocorrelation coefficients

cates that the duration time for scale change into the smaller one is very short in comparison with time scale of the turbulent fluctuation. This fact and the nonexistence of a corresponding reversed change in the cycle strongly suggest that the turbulence generates at $\omega t=\pi / 4$.

Fig. 9 shows the energy spectra $E(n)$, which are obtained by using a Fourier transformation of the correlation function at $\omega t=\pi / 4$. They correspond to the two regions, i. e. $t^{\prime}>0$ and $t^{\prime}<0$, of the correlation coefficient. Different from a steady-flow spectrum, which decreases with a gentle slope as the frequency increases, they show a selectivity of fre-

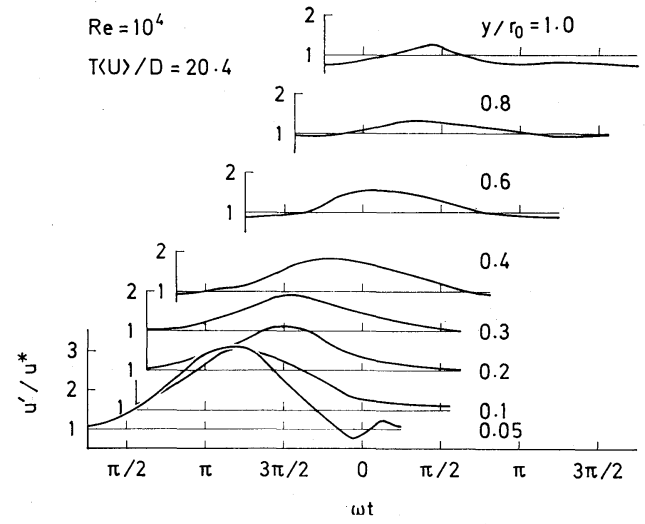

Fig. 11 Variations of turbulence intensity after rapid change of time scale

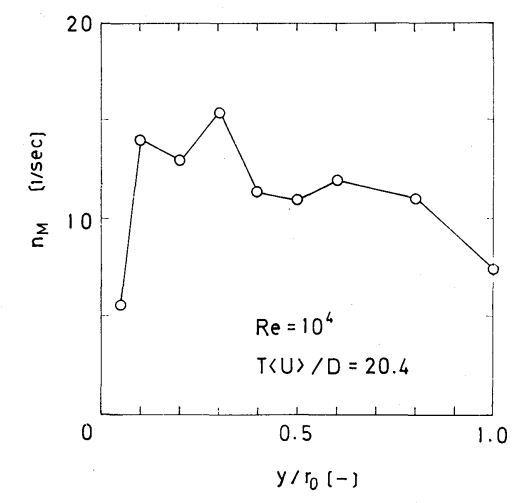

Fig. 12 Medium frequency of propagating turbulence

quency $n$, and selective frequencies of the two spectra are complementary to each other. Thus, at the instance of generation, turbulence which consists of selective components of frequency changes to the complementary constitution.

\section{4 Propagation of generated turbulence}

Next, propagation of the generated turbulence will be studied. An asymmetrical correlation curve, which represents a rapid change of scale, was also found at $y^{+} \geqslant 15.7$. Fig. 10 shows the most asymmetrical correlation curves at different radial positions. It occurs at later phase but less conspicuously with increasing radial distance from the wall; the difference of phase is indicated in the same figure as the time period $\overline{\Delta t}$.

Fig. 11 shows a variation of intensity of turbulence from the instance of the rapid change of scale at each radial point. Although maximum value decreases as the distance from the wall increases, a similar variation appears throughout the cross-section of the tube and the phase difference of the maximum intensity is nearly equal to that of the rapid change of scale.

From the above results it is clear that, decreasing its coherency, the generated turbulence propagates to the centre-line of the tube.

Fig. 12 shows the change of medium frequency 
$n_{M}$ during the propagation. It becomes high at $y / r_{0}=$ 0.1 and then decreases slowly. So it can be said that the propagation process is not a monotonically diffusive one but is affected by some discrete events occurring in sequence.

In fact, the changes of propagation time and medium frequency indicate that the process is made up by three stages: (i) the generated turbulence propagates slowly to $y / r_{0} \simeq 0.1$ or $y^{+} \simeq 31.4$; (ii) it changes to high frequency and propagates quickly to $y / r_{0} \simeq 0.3$ or $y^{+} \simeq 94.3$ and then, (iii) becoming of lower frequency slowly it propagates to $y / r_{0}=1.0$ with nearly constant propagation speed. It is apparent that the 'appearance of a relatively low speed region of fluid' occurs in the first stage and the 'lift-up' in the second stage. However, the temporal characteristics ${ }^{2)}$ of the 'oscillatory growth' and the 'breakup' do not appear in the propagation process. The variation of intensity of turbulence suggests that the breakup subsequent to the oscillatory growth occurs near the wall at $t \simeq 0.35 \mathrm{sec}$, when the generated turbulence propagates to $y / r_{0} \simeq 0.6$ or $y^{+} \simeq 188$. Thus it is clear that the turbulence which propagates is not the one associated with the lifted-up fluid which shows oscillatory growth and the breakup of turbulence in the wall region $\left(0<y^{+}<70\right)$; the propagation is considered to be associated with the large-scale vortex which was observed by Nychas et al. ${ }^{5)}$ and Offen and Kline ${ }^{6)}$.

\section{5 Mean propagation time $\overline{\Delta t}$}

The radial distribution of mean propagation time for four pulsation periods are shown in Fig. 13. As can be seen, they are independent of pulsation period; that is, they are not affected by the change of axial velocity.

Moreover, it is worth noting that the value of mean propagation time at the centre-line of the tube is nearly equal to the mean burst period $\left(0.53 \mathrm{sec}\right.$ at $\left.R e=10^{4}\right)$. This was confirmed at other Reynolds numbers. Fig. 14 shows the mean propagation time elapsed from the generation instance at $y^{+}=15.7$. The values decrease rapidly with increasing Reynolds number, and each value at $y / r_{0}=1$ agrees well with mean burst period. Accordingly, if the pulsation period is shorter than the mean burst period, the propagation does not reach the centre-line of the tube during one pulsation period, and so the periodical change of intensity of turbulence is presumed to appear only in the limited region near the wall, as mentioned before.

Further, the facts that the mean propagation time is not affected by the pulsation and that its value at the centre-line of the tube is equal to the mean burst period strongly suggest that the propagation process is consistent not only in pulsating flow but also in steady flow.

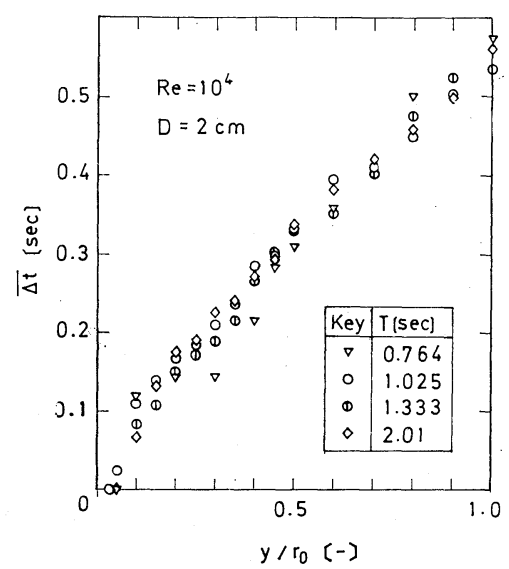

Fig. 13 Mean propagation time as a function of $y / r_{0}$ and $T$

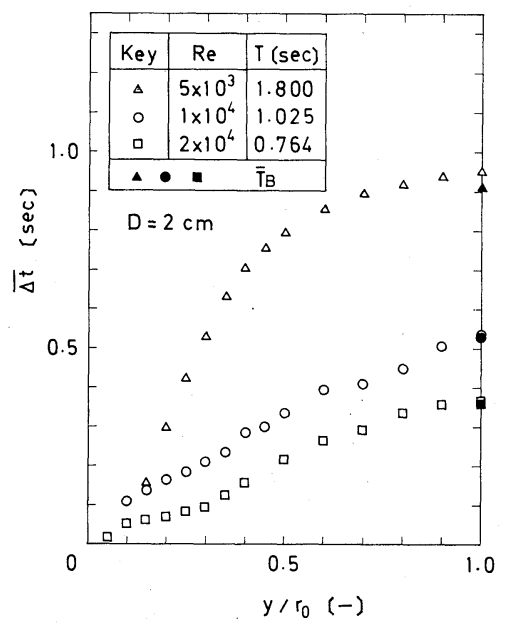

Fig. 14 Mean propagation time as a function of $y / r_{0}$ and and Reynolds number

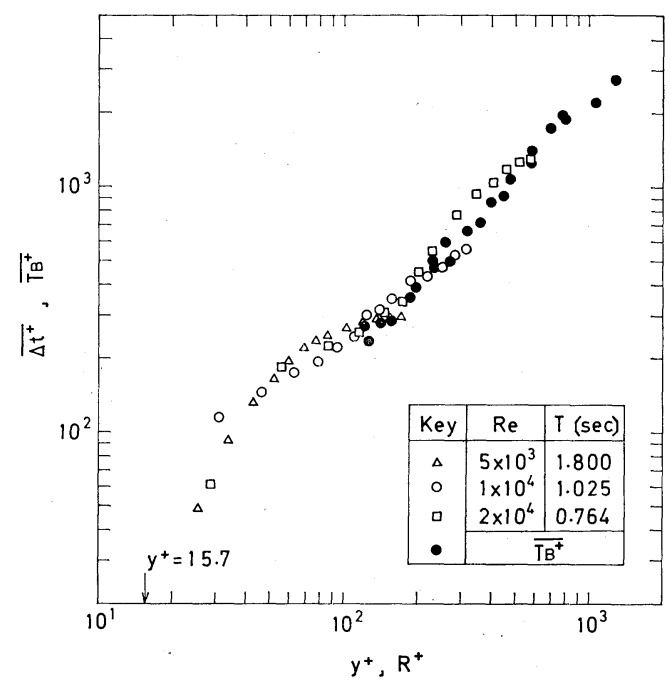

Fig. $15 \overline{\Delta t}$ and $\overline{T_{B}}$ normalized with wall parameters as functions of $\boldsymbol{y}^{+}$and $\boldsymbol{R}^{+}$

\section{6 Mean burst period}

Fig. 15 shows the mean propagation time normalized by the wall parameters $\nu$ and $u^{*}$ as a function of $y^{+}$. As can be seen, there is an excellent correlation of results for all Reynolds numbers, i. e., a law of the 
wall is applicable to the propagation time, and the values of $\overline{\Delta t}^{+}$are in good agreement with those of ${\overline{T_{B}}}^{+}$which are plotted against dimensionless radius $R^{+}$.

Accordingly, it can be said that the entire cycle of bursting is characterized by the propagation process and that the bulk parameter dependence of mean burst period is attributed to the propagation distance, which is nearly equal to pipe radius.

Further, the explanation of the periodicity of bursting will be completed, if the last stage of propagation is linked to the first stage or generation of turbulence. To this end, as already mentioned, the rapid change of the selective frequencies to the complementary values at the instance of generation is considered to be one of the useful clues. At the same time, however, it must be noted that there may be a clear difference between the generation of turbulence in steady flow and that in resonant pulsating flow where the generation is ruled directly by the pulsating velocity.

\section{Conclusion}

1. In the preferred range of burst period, the turbulence is generated near the wall by the flow pulsation and at the instance of generation, the frequencies of turbulence become selective and change to the complementary value.

2. Decreasing its coherency, the generated turbulence propagates radially to the centre-line of the tube. The mean propagation time is independent of the pulsation period and scales on the wall parameters.

3. The mean propagation time, in which the turbulence propagates from its origin to the centre-line of the tube, agrees well with the mean burst period of steady turbulent flow. This fact strongly suggests that the entire cycle of bursting is characterized by the propagation of generated turbulence in the radial direction and that the bulk parameter dependence of mean burst period is attributed to the pipe radius.

4. Due to this constant time of propagation, the periodical change of intensity of turbulence becomes to be localized within near the wall with decreasing pulsation period in the region $T_{B_{m i n}}<T<\overline{T_{B}}$.

\section{Nomenclature}

$D \quad=$ pipe diameter

$[\mathrm{cm}]$

\begin{tabular}{|c|c|c|}
\hline$E(n)$ & $=$ one-dimensional energy spectrum & {$\left[\mathrm{cm}^{2} / \mathrm{sec}\right]$} \\
\hline$N$ & $\begin{aligned}= & \text { number of cycles evaluated for each } \\
& \text { value of } U(t) \text { and } u^{\prime}(t)\end{aligned}$ & {$[-]$} \\
\hline$n$ & $=$ frequency & {$[1 / \mathrm{sec}]$} \\
\hline$n_{M}$ & $=$ medium frequency, $\int_{0}^{n_{M}} E(n) /\left(u^{\prime}\right)^{2} d n=0.5$ & {$[1 / \mathrm{sec}]$} \\
\hline$R^{+}$ & $=$dimensionless radius $\left(=r_{0} u^{*} / \nu\right)$ & {$[-]$} \\
\hline $\operatorname{Re}$ & $=$ Reynolds number $(=D\langle U\rangle / \nu)$ & {$[-]$} \\
\hline$R_{u u}\left(t, t^{\prime}\right)$ & $\begin{aligned}= & \text { phase-averaged autocorrelation } \\
& \text { function, Eqs. (3) and (4) }\end{aligned}$ & {$[-]$} \\
\hline$r_{0}$ & $=$ radius of circular tube & [cm] \\
\hline$T$ & $=$ period of pulsation & [sec] \\
\hline$T_{B}$ & $=$ burst period & [sec] \\
\hline$\overline{T_{B}}$ & $=$ mean value of $T_{B}$ & [sec] \\
\hline$\overline{T_{B}}+$ & $=$ dimensionless period $\left(=\overline{T_{B}}\left(u^{*}\right)^{2} / \nu\right)$ & {$[-]$} \\
\hline$T_{B_{\min }}$ & $=$ minimum value of $T_{B}$ & [sec] \\
\hline$T_{c}$ & $=$ critical period of pulsation & [sec] \\
\hline$t$ & $=$ time & [sec] \\
\hline$t^{\prime}$ & $=$ delay time & [sec] \\
\hline$\Delta t$ & $\begin{aligned}= & \text { time interval of propagation of } \\
& \text { generated turbulence }\end{aligned}$ & [sec] \\
\hline$\overline{\Delta t}$ & $=$ mean value of $\Delta t$ & [sec] \\
\hline$\overline{\Delta t}^{+}$ & $=$dimensionless time $\left(=\overline{\Delta t}\left(u^{*}\right)^{2} / \nu\right)$ & {$[-]$} \\
\hline$U$ & $=$ local velocity in axial direction & {$[\mathrm{cm} / \mathrm{sec}]$} \\
\hline$\langle U\rangle$ & $\begin{array}{l}=\text { velocity obtained by averaging the } \\
\text { value } U \text { over the cross-section }\end{array}$ & {$[\mathrm{cm} / \mathrm{sec}]$} \\
\hline$U_{i}$ & $=$ instantaneous value of $U$ & {$[\mathrm{~cm} / \mathrm{sec}]$} \\
\hline$u$ & $\begin{array}{l}=\text { fluctuating component of velocity in } \\
\text { axial direction }\end{array}$ & {$[\mathrm{cm} / \mathrm{sec}]$} \\
\hline$u^{\prime}$ & $=$ root-mean square of $u$ & {$[\mathrm{~cm} / \mathrm{sec}]$} \\
\hline$u^{*}$ & $\begin{aligned}= & \text { friction velocity in steady flow } \\
& \left(=\sqrt{\left.\tau_{W} / \rho\right)}\right.\end{aligned}$ & {$[\mathrm{cm} / \mathrm{sec}]$} \\
\hline$y$ & $=$ normal distance from wall & [cm] \\
\hline$y^{+}$ & $=$dimensionless distance $\left(=y u^{*} / \nu\right)$ & {$[-]$} \\
\hline$\nu$ & $=$ kinematic viscosity & {$\left[\mathrm{cm}^{2} / \mathrm{sec}\right]$} \\
\hline$\rho$ & $=$ density & {$\left[\mathrm{g} / \mathrm{cm}^{3}\right]$} \\
\hline$\tau_{W}$ & $=$ shear stress at wall in steady flow & $\left.\mathrm{s} / \mathrm{cm}^{2} \cdot \mathrm{sec}^{2}\right]$ \\
\hline$\omega$ & $=$ angular frequency & {$[1 / \mathrm{sec}]$} \\
\hline
\end{tabular}

\section{Literature Cited}

1) Corino, E. R. and R. S. Brodkey: J. Fluid Mech., 37, 1 (1969).

2) Kim, H. T., S. J. Kline and W. C. Reynolds: ibid., 50, 133 (1971).

3) Mizushina, T., T. Maruyama, S. Ide and Y. Mizukami: $J$. Chem. Eng. Japan, 6, 152 (1973).

4) Mizushina, T., T. Maruyama and Y. Shiozaki: ibid., 6, 487 (1973).

5) Nychas, S. G., H. C. Hershey and R. S. Brodkey: J. Fluid Mech., 61, 513 (1973).

6) Offen, G. R. and S. J. Kline: ibid., 62, 223 (1974).

7) Pennell, W. T., E. M. Sparrow and E. R. G. Eckert: Int. J. Heat Mass Transfer, 15, 1067 (1972).

8) Rao, K. N., R. Narasimha and M. A. Badri Narayanan: J. Fluid Mech., 48, 339 (1971). 